\title{
Coexistence of Ballistic and Fourier Regimes in the $\beta$ Fermi-Pasta-Ulam-Tsingou Lattice
}

\author{
Giovanni Dematteis $\odot,{ }^{1, *}$ Lamberto Rondoni $\odot,{ }^{2}$ Davide Proment $\odot,{ }^{3}$ Francesco De Vita $\odot,{ }^{1}$ and Miguel Onorato $\odot^{1,4}$ \\ ${ }^{1}$ Dipartimento di Fisica, Università degli Studi di Torino, Via Pietro Giuria 1, 10125 Torino, Italy \\ ${ }^{2}$ Dipartimento di Scienze Matematiche, Politecnico di Torino, Corso Duca degli Abruzzi 24, I-10129 Torino, Italy \\ ${ }^{3}$ School of Mathematics, University of East Anglia, Norwich Research Park, NR47TJ Norwich, United Kingdom \\ ${ }^{4}$ INFN, Sezione di Torino, Via Pietro Giuria 1, 10125 Torino, Italy
}

(Received 20 December 2019; revised 19 May 2020; accepted 5 June 2020; published 7 July 2020)

\begin{abstract}
Commonly, thermal transport properties of one-dimensional systems are found to be anomalous. Here, we perform a numerical and theoretical study of the $\beta$-Fermi-Pasta-Ulam-Tsingou chain, considered a prototypical model for one-dimensional anharmonic crystals, in contact with thermostats at different temperatures. We give evidence that, in steady state conditions, the local wave energy spectrum can be naturally split into modes that are essentially ballistic (noninteracting or scarcely interacting) and kinetic modes (interacting enough to relax to local thermodynamic equilibrium). We show numerically that the well-known divergence of the energy conductivity is related to how the transition region between these two sets of modes shifts in $k$ space with the system size $L$, due to properties of the collision integral of the system. Moreover, we show that the kinetic modes are responsible for a macroscopic behavior compatible with Fourier's law. Our work sheds light on the long-standing problem of the applicability of standard thermodynamics in one-dimensional nonlinear chains, testbed for understanding the thermal properties of nanotubes and nanowires.
\end{abstract}

DOI: 10.1103/PhysRevLett.125.024101

Deriving equations for the macroscopic observables from microscopic dynamics is the core of statistical physics. Often, such "reduction" passes through an intermediate stochastic model for the mesoscopic scale, c.f. the celebrated Boltzmann equation for a rarefied gas [1], used to derive the hydrodynamic equations (e.g., Euler, or Navier-Stokes) [2]. Linear response theory is a sound framework to compute the macroscopic transport coefficients [3-5]. In a solid rod whose ends are kept at not excessively different temperatures one expects Fourier's law of heat conduction to hold and the Green-Kubo formula to yield the conductivity as an integral of the time correlation of the heat current at equilibrium [3]. This program has been pursued for one-dimensional (1D) systems [6-8], typically modeled as lattices of point particles interacting via springlike forces. In fact, 1D structures such as nanowires and nanotubes have become available in lab experiments, with widespread applications in industry and technology [9-13], making an understanding of 1D transport of primary importance. Thus, harmonic [6] as well as anharmonic lattices have been widely studied $[8,14]$. Nevertheless, establishing the macroscopic equations for low-dimensional structures results challenging $[8,14]$; the dimensional constraints imply a slow decay of correlations that may prevent the convergence of the GreenKubo integral. The approach based on the wave kinetic equation, i.e., the phonon Boltzmann equation of solid state physics and main object of wave turbulence theory [15], has recently opened an important perspective in this field
[16-19]. The wave kinetic equation concerns phonons that interact with each other through resonant $n$-wave collisions, providing an effective relaxation mechanism toward thermodynamic equilibrium [19-22]. Although the heat conductivity can be computed rigorously $[17,18,23]$ when a pinning onsite potential is introduced, for unpinned anharmonic lattices energy conduction appears nontrivially anomalous $[8,14,16,24-26]$. The usual procedure is to introduce a heuristic cutoff in the Green-Kubo integral based on the sound speed [14,18], but a rigorous justification is still lacking [8]. Moreover, there is no clear interpretation of the Green-Kubo formula in cases where the deviations from local thermodynamic equilibrium are significant [27-33]. Mainly due to these difficulties, the derivation of proper macroscopic equations for such systems remains a long-standing open problem.

In this Letter, we use concepts of wave-kinetic theory to investigate the low-temperature regime of the $\beta$ FermiPasta-Ulam-Tsingou model ( $\beta$-FPUT) [34-37], paradigmatic anharmonic 1D lattice. In the thermodynamic limit, the mechanism of thermalization at the mesoscopic scale is related to four-wave resonant interactions [22]. We give evidence from direct numerical simulations that the system splits into two independent sets of modes: the low- $k$ modes, with the mean free path exceeding what we call the "mesoscopic" scale $\lambda$, and the remaining modes that, interacting resonantly, relax to local thermodynamic equilibrium. We show that the high- $k$ modes transport energy diffusively, hence the anomalous-transport scaling is due to 
how the wave numbers associated with the transition modes (not diffusive but also not perfectly ballistic) scales with $L$. More precisely, we give evidence that (i) our scaling of the transition between ballistic and kinetic modes dictates the scaling of the energy conductivity with the size $L$; (ii) the conductivity restricted to the kinetic modes converges, as in standard heat conduction; and (iii) the energy density is dominated by the locally thermalized kinetic modes, implying an apparently regular Fourier temperature profile. Results are supported by extensive numerical simulations and a dimensional argument applied on the wave kinetic equation.

The $\beta$-FPUT system is defined by the Hamiltonian $\mathcal{H}=\mathcal{H}_{h}+\mathcal{H}_{a}$ with

$$
\begin{aligned}
\mathcal{H}_{h} & =\sum_{j=1}^{N} \frac{1}{2} p_{j}^{2}+\frac{1}{2}\left(q_{j}-q_{j-1}\right)^{2}, \\
\mathcal{H}_{a} & =\frac{\beta}{4} \sum_{j=1}^{N}\left(q_{j}-q_{j-1}\right)^{4} .
\end{aligned}
$$

Let $J_{e}$ be the (harmonic) energy current per particle,

$$
J_{e}=\frac{1}{N} \sum_{j=1}^{N} \frac{1}{2}\left(p_{j}+p_{j-1}\right)\left(q_{j}-q_{j-1}\right),
$$

$T_{ \pm}=\bar{T} \pm \Delta T / 2$ the temperatures of the thermostats at the two ends of the $\beta$-FPUT chain, where $N$ is the number of particles of unitary mass and unitary lattice spacing $(N=L)$. Let us assume $\Delta T \ll \bar{T}$ and consider the lowtemperature regime, so that the ratio of the anharmonic and the harmonic parts of the Hamiltonian is small, $\mathcal{H}_{a} / \mathcal{H}_{h} \simeq \beta \bar{T} \ll 1$. For lattices, it is standard to define the thermal conductivity as

$$
\kappa_{e}=\left\langle J_{e}\right\rangle N / \Delta T,
$$

where $\langle\cdot\rangle$ indicates canonical ensemble average, assuming that the system is close to equilibrium at temperature $\bar{T}$. For normal heat conduction $\kappa_{e}$ tends to a constant as $N \rightarrow \infty$, while transport is called anomalous if $\kappa_{e}$ diverges, e.g., as $N^{\delta}, \delta>0$. For the harmonic chain $(\beta=0)$ the problem was solved analytically in [6]: the conductivity is proportional to $N(\delta=1)$. Such transport behavior, with no temperature gradient arising in the chain bulk (since wave packets, or phonons, do not interact), is called ballistic. On the other hand, for the $\beta$-FPUT chain an exponent $\delta \simeq 0.4$ was found by some authors, while others proposing $\delta=1 / 2$ or $\delta=1 / 3[14]$.

In the wave-turbulence formalism $[15,17,22,23]$ we use the normal variable $a_{k}:=\left(\omega_{k} q_{k}+i p_{k}\right) / \sqrt{2 \omega_{k}}$, with $\omega_{k}=2|\sin (k / 2)|$, and the wave-action spectral density associated with $a_{k}, n_{k}=n(k, x, t)$. One can derive, although not fully rigorously, the following wave kinetic equation $[15-20,26]$ :

$$
\frac{\partial n_{k}}{\partial t}+v_{k} \frac{\partial n_{k}}{\partial x}=I_{k}
$$

The collision integral $I_{k}$ is conveniently split as a difference of two terms: $I_{k}=\eta_{k}-n_{k} / \tau_{k}$, with

$$
\begin{aligned}
\eta_{k_{1}}= & 4 \pi \int_{-\pi}^{\pi}\left|T_{1234}\right|^{2} n_{k_{2}} n_{k_{3}} n_{k_{4}} \delta\left(k_{12}^{34}\right) \delta\left(\omega_{12}^{34}\right) d k_{2} d k_{3} d k_{4} \\
\frac{1}{\tau_{k_{1}}}= & 4 \pi \int_{-\pi}^{\pi}\left|T_{1234}\right|^{2}\left(-n_{k_{3}} n_{k_{4}}+n_{k_{2}} n_{k_{3}}+n_{k_{2}} n_{k_{4}}\right) \\
& \times \delta\left(k_{12}^{34}\right) \delta\left(\omega_{12}^{34}\right) d k_{2} d k_{3} d k_{4} \\
\left|T_{1234}\right|^{2}= & \frac{9}{16} \omega_{k_{1}} \omega_{k_{2}} \omega_{k_{3}} \omega_{k_{4}}
\end{aligned}
$$

in a spatial domain $x \in[0, L]$, using the notation: $y_{12}^{34}:=y_{k_{1}}+y_{k_{2}}-y_{k_{3}}-y_{k_{4}}$. The group velocity is denoted by $v_{k}=d \omega_{k} / d k$. The second term in the lhs of Eq. (4) quantifies advection due to spatial inhomogeneities. $\tau_{k}$ can be interpreted as the mean collision time of mode $k$.

In the harmonic chain, the collision integral is missing and Eq. (4) predicts ballistic advection of wave action carried by harmonic excitations with speed $v_{k}$. The rhs of (4) is the four-wave collision integral, and represents the effective mechanism of relaxation to equilibrium [22]. Note that, for finite $N$ and small nonlinearity, six-wave resonant interactions dominate $[19,20,38]$, but we made sure that $N$ in our simulations is large enough for the Fourier space to be sufficiently dense, thereby making four-wave interactions dominate [22].

Now, $n_{k}$ must be a slowly varying function of $x$ : Eq. (4) assumes the existence of a mesoscopic scale, $\lambda$, that is much smaller than the macroscopic scale. This suggests an operative definition of $\lambda$ as the largest size over which the system can be considered approximately spatially homogeneous, i.e., as the largest scale on which the use of the Fourier transform is justified. When the system is thermodynamic, the characteristic microscopic interaction distance is finite. Thus, a mesoscopic scale $\lambda$ within which practically all phonons interact, and relaxation takes place, can be identified [4]. Moreover, boxes of size $\lambda$ appear as points of a continuum in the $\lambda / L \rightarrow 0$ limit. In short, considering a partition of the system consisting of $L / \lambda$ adjacent boxes, two limits need to be taken: (i) $L \rightarrow \infty$ at fixed boundary temperatures, so that the object is large compared to the microscopic scales and a continuum description makes sense; (ii) $\lambda \rightarrow \infty$, which yields the continuous $k$-space formalism (thermodynamic limit) of (4), at fixed $x$. In principle, there are different ways of combining these limits, that correspond to different physical situations. In our theoretical argument, we will assume that the separation between the mesoscale and the macroscale is of one order of magnitude, i.e., $L \propto \lambda^{2}$; such choice corresponds to the mesoscopic scale being placed in between the microscopic and the macroscopic scales (cf. the 
hydrodynamic diffusive scaling of the Boltzmann equation $[2,39])$. We will give evidence that our results, obtained integrating numerically the $\beta$-FPUT equation of motion, are consistent with our assumption.

Having defined the scales, we focus our attention on the wave kinetic Eq. (4). In stationary conditions, there are two competing contributions: the collision integral and the transport term. In order to observe regular heat conduction, we expect the collision term to guarantee local relaxation to thermodynamic equilibrium within any homogeneous subdomain of width $\lambda$. On the other hand, if the collision integral is not active enough, energy flows in a ballistic way from one subdomain to the next one, without having been thermalized. As observed in our numerical simulations, the situation for the $\beta$-FPUT system is hybrid: the collision integral is able to make the system thermalize locally up to some critical wave number $k_{c}$, while lower $k$ waves scarcely interact and carry energy almost ballistically between adjacent subdomains. Using a dimensional argument, the scaling of $k_{c}$ with $L$ can be estimated from the wave kinetic equation assuming that, in stationary conditions, the transport term and the collision integral balance:

$$
\frac{v_{k_{c}}}{\lambda} \sim \frac{1}{\tau_{k_{c}}} .
$$

Assuming very small deviations from local equilibrium (see discussion at the end of the Letter) and small $k$, a direct analytical calculation $[16,26]$ yields

$$
\tau_{k} \propto k^{-5 / 3}, \quad \text { for }|k| \ll 1,
$$

which plugged into Eq. (6) leads to

$$
k_{c} \propto \lambda^{-3 / 5} \propto L^{-3 / 10},
$$

where we have used the fact that $L \propto \lambda^{2}$ and, for small $k$, $v_{k} \simeq 1$. Based on the above discussion, we conjecture that (i) the field $a_{k}$ can be split into two parts: the modes with $|k|>k_{c}$ are essentially in thermodynamic equilibrium within cells of size $\lambda$ and verify Fourier's law, while for $|k| \leq k_{c}$ the modes are ballistic or scarcely interacting; (ii) the scaling of $\kappa_{e}$ with $L$ is strictly related to the scaling of $k_{c}$ with $L$, Eq. (8). Numerical evidence of the conjecture is given below.

Our simulations solve $2 N$ coupled equations of motion for the Hamiltonian (1) with $\beta=0.1$, integrated with fourth order Runge-Kutta method with time step $5 \times 10^{-2}$. Fixed boundary conditions are used for the two walls $q_{0}=0$ and $q_{N+1}=0$, and the two particles $j=1$ and $j=N$ are coupled with Nosé-Hoover thermostats at temperatures $T_{+}=0.3$ and $T_{-}=0.2$, respectively, at the left and the right ends. In all our simulations we consider $N$ sufficiently large so that the discrete- $k$ effects, which lead to a lack of

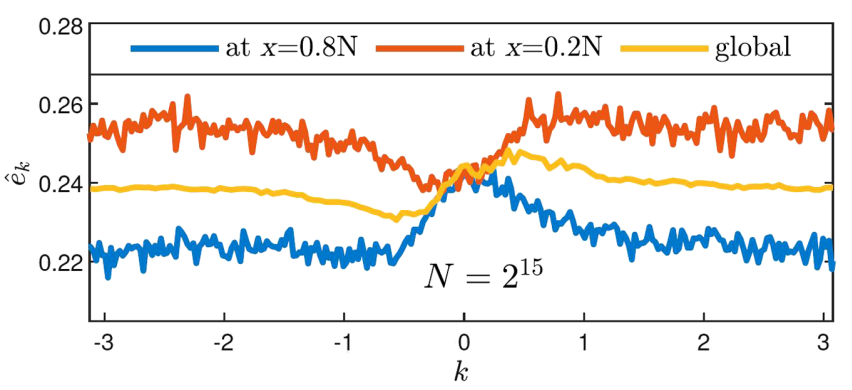

FIG. 1. Steady state energy spectrum for $N=2^{15}$.

four-wave resonances, do not take place, see [22]. Differently from previous works where the spectral analysis is performed on the full length of the chain, we are interested in the local spectral properties of the system. Therefore, we consider the mesoscopic length $\lambda=\sqrt{N}=\sqrt{L}$. Macroscopic observables are averaged spatially over mesoscopic boxes of size $\lambda$, and in time at the steady state, over $2.5 \times 10^{5}$ time units after an initial relaxation transient of $5 \times 10^{4}$ units. An additional ensemble averaging over five independent realizations is used to improve statistical convergence. In Fig. 1, we show the wave energy spectral density (energy spectrum), $\hat{e}_{k}=\omega_{k} n_{k}$, computed locally on a spatial window of mesoscopic size, around two points placed at $x=0.2 L$, close to the thermostat at higher temperature and $x=0.8 L$, close to the one at lower temperature. Observe that the energy spectrum is clearly asymmetric for small $k$. Moreover, for high wave numbers the spectrum is almost flat, i.e., those modes are in an equipartition state. In order to highlight local thermalization, noticing that the asymmetric part of the spectrum does not contribute to the total energy, in Fig. 2 we plot the symmetrized energy spectrum $\left(\hat{e}_{k}+\hat{e}_{-k}\right) / 2$, for different values of $N$. Local equipartition is observed for the high wave numbers, say the modes with $|k|>k_{c}$. Instead, the energetic content of the low wave number modes $\left(|k|<k_{c}\right)$ tends to the average temperature $\bar{T}$ throughout the chain. As $N$ increases, $k_{c}$ shifts toward the origin and the separation between the two states becomes sharper. For $N=2^{15}$, the two states are clearly separated with a narrow transition region.

In Fig. 3 we show the energy per particle $e(x)$, averaged in time and in space over mesoscopic boxes of width $\lambda=$ $\sqrt{N}$ for $N=2^{9}$ and $N=2^{13}$. The figure shows that as $N$ increases the profile tends to the expected Fourier profile. In the lower panel, the case for $N=2^{13}$ is further investigated and $e(x)$ is decomposed as

$$
e(x)=e^{>}\left(x ; k_{c}\right)+e^{<}\left(x ; k_{c}\right),
$$

using the estimate $k_{c}=0.75$ from the central panel of Fig. 2, and where $e^{>}\left(x ; k^{\prime}\right)$ is obtained from $q(x)$ and $p(x)$ after they have been filtered in Fourier space and only contributions from wave numbers $|k|>k^{\prime}$ have been retained; $e^{<}\left(x ; k^{\prime}\right)$ has contributions only from $|k| \leq k^{\prime}$. 

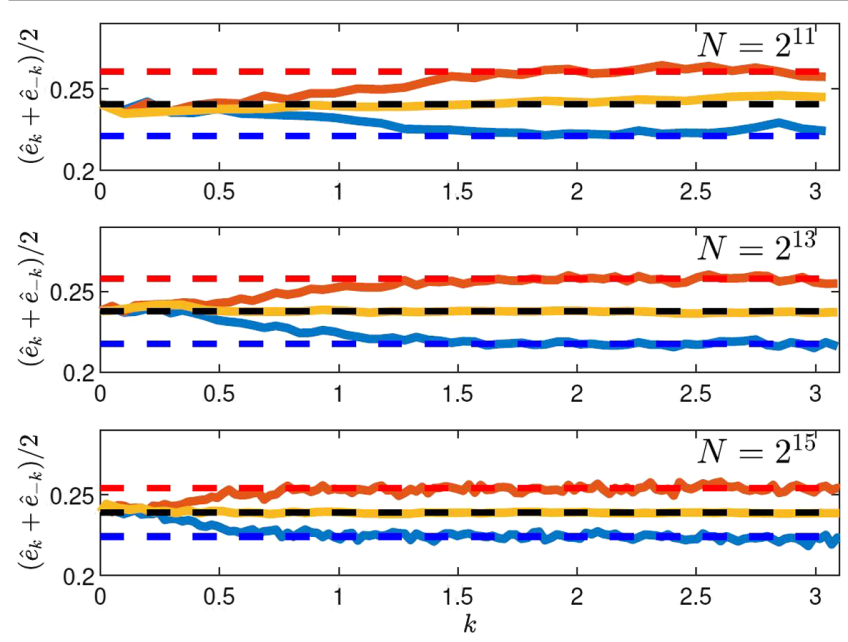

FIG. 2. The red and blue solid lines are the symmetrized stationary local energy spectra computed in windows of width $\sqrt{N}$ centered at $x_{1}=0.2 N$ and $x_{2}=0.8 N$, respectively. The dashed lines with same colors are at the respective average energy per particle at $x_{1}$ and $x_{2}$. The yellow line is the spectrum performed on the full chain.

As conjectured, the profile for $e^{>}\left(x ; k_{c}\right)$ is consistent with the expected linear profile, typical of Fourier's law, and the second one is consistent with a flat profile with temperature $\bar{T}$; this latter behavior is typical of harmonic (collisionless) chains.
We now devote our attention to the energy fluxes; in steady state conditions the flux is independent of $x$. We therefore define the net spectral energy current [7], as

$$
\hat{j}_{e}(k):=v_{k} \omega_{k}\left(n_{k}-n_{-k}\right) / 2,
$$

which depends on the asymmetric part of the spectrum. Note that $\hat{j}_{e}(k)=\hat{j}_{e}(-k)$ and the Fourier transform for calculating $n_{k}$ is made on the entire length of the chain. This is because in stationary conditions the flux is independent of $x$. In Fig. 4, the upper panels show the behavior of $\hat{j}_{e}(k)$ while varying $N$. As $N$ doubles, $\hat{j}_{e}$ remains constant for low wave numbers (ballistic modes), while it roughly halves for higher wave numbers (kinetic modes). This means that the ballistic-modes contribution to the energy current is independent of $N$, as expected for the harmonic chain [6], while for the kinetic modes it is proportional to $N^{-1}$. In the lower left panel we show the energy conductivity as defined by Eq. (3), where $\left\langle J_{e}\right\rangle=\sum_{k} \hat{j}_{e}(k)$. The plot shows a scaling compatible with previous numerical results, $\kappa_{e} \sim L^{2 / 5}$. We now consider the filtered conductivity

$$
\kappa_{e}^{>}(k)=\frac{N}{\Delta T} \sum_{\left|k^{\prime}\right|>k} \hat{j}_{e}\left(k^{\prime}\right), \kappa_{e}^{<}(k)=\frac{N}{\Delta T} \sum_{\left|k^{\prime}\right|<k} \hat{j}_{e}\left(k^{\prime}\right),
$$
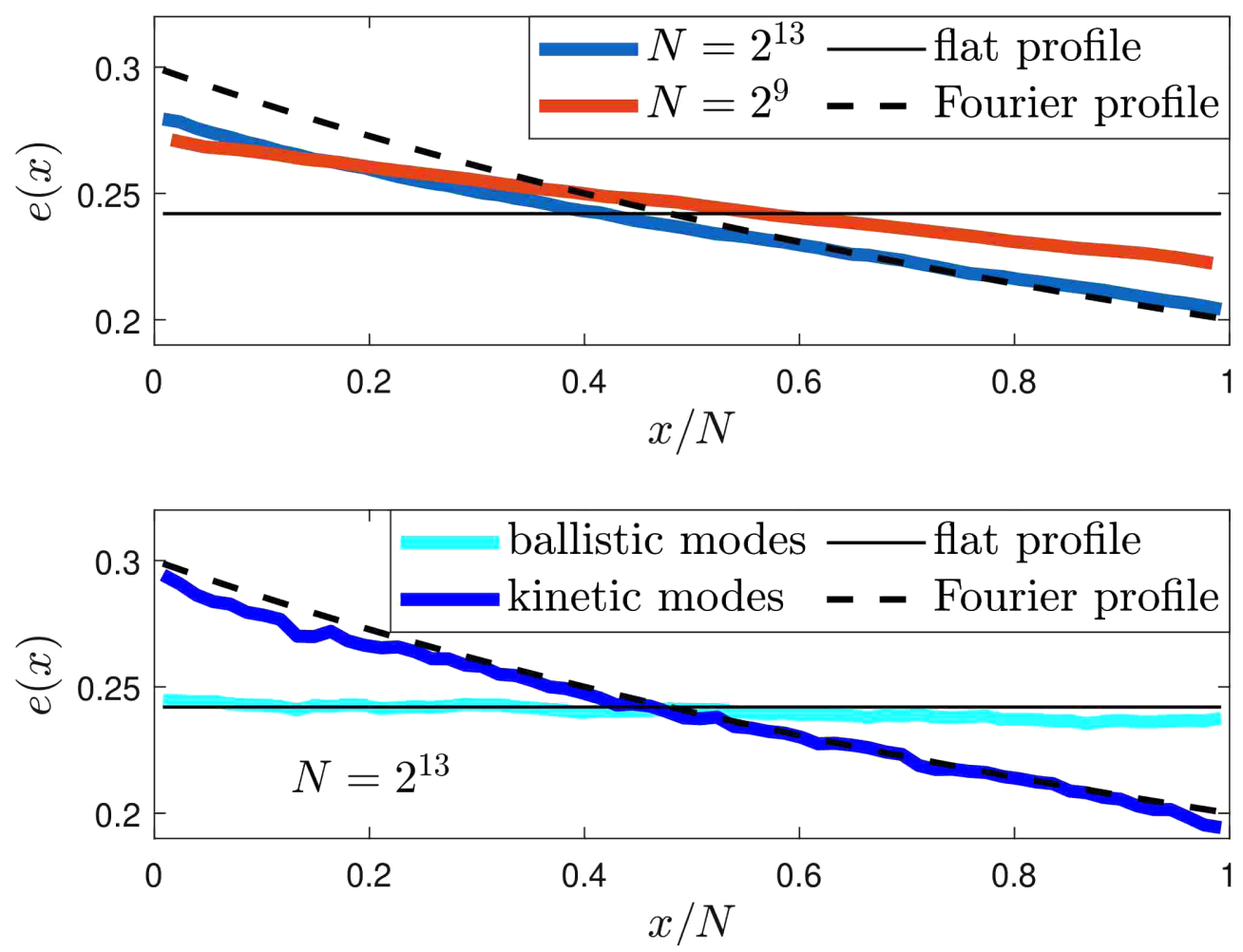

FIG. 3. Top: Numerical profiles of $e(x)$. Bottom: the blue line of the upper panel $\left(N=2^{13}\right)$ is decomposed into its contributions from $|k| \leq k_{c}$ (light blue) and $|k|>k_{c}$ (dark blue), normalized by the fraction of modes in each set: $k_{c} / \pi$ and $\left(\pi-k_{c}\right) / \pi$, respectively. A value $k_{c}=0.75$ is used, based on numerical estimate from Fig. 2. 

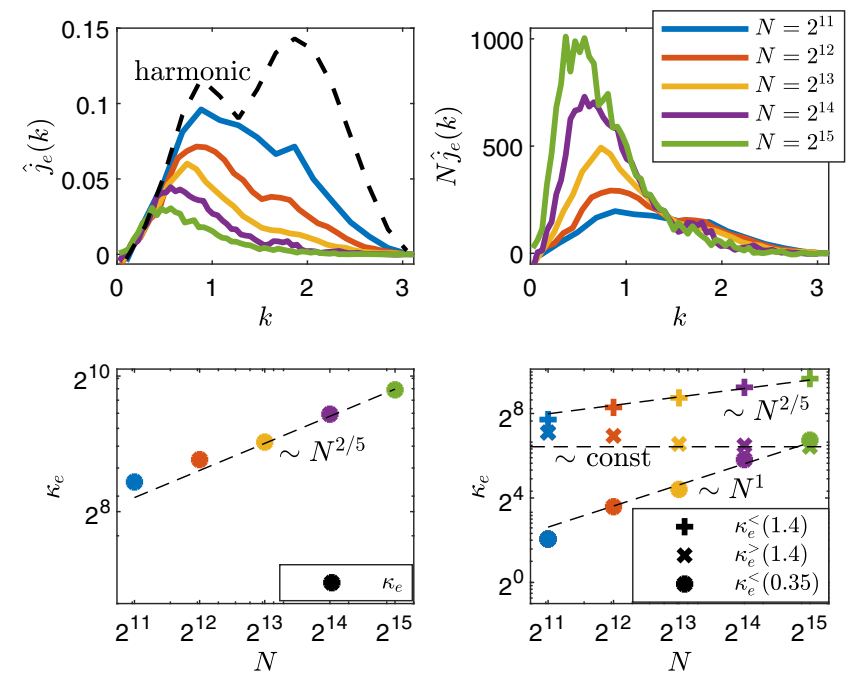

FIG. 4. Top left: net average energy current per mode $\hat{j}_{e}(k)$. Top right: scaled current $N \hat{j}_{e}(k)$ (cf. Fig. 2.6 of [40]). Bottom left: $N$-dependent scaling of $\kappa_{e}$ compared to the $N^{2 / 5}$ scaling (black dashed line). Bottom right: $N$-dependent scaling behavior for the filtered conductivity: ballistic $\mathrm{O}(N)$ divergence at low $k \mathrm{~s}$, kinetic constant conductivity at high $k \mathrm{~s}$, and $\mathrm{O}\left(N^{2 / 5}\right)$ divergence.

with $\kappa_{e}=\kappa_{e}^{<}(k)+\kappa_{e}^{>}(k)$. On the lower right panel of Fig. 4 we show three quantities with different scalings: choosing a threshold $k=1.4>k_{c}$ for all $N$ (cf. Fig. 2), we observe that $\kappa_{e}^{<}(1.4) \sim N^{2 / 5}$, while $\kappa_{e}^{>}(1.4)$ converges to constant (compatible with regular Fourier's law). On the other hand, considering wave numbers that are always ballistic $\left(0.35<k_{c}\right.$ for all $\left.N\right)$, thus excluding modes around $k_{c}$, we obtain that $\kappa_{e}^{<}(0.35) \sim N^{1}$, typical of the harmonic chain but far from the anomalous exponent 0.4. Thus, the way $k_{c}$ scales with the size $L$, Eq. (8), plays a key role concerning the anomaly, as will be shown in the following. Multiplying (4) by $\omega_{k}$ and integrating over $k \in[-\pi, \pi]$ yields

$$
\partial_{\tau} e(x, \tau)=-\partial_{x} j_{e}(x, \tau),
$$

where $e(x, \tau):=\int_{-\pi}^{+\pi} \hat{e}(k) d k$ and $j_{e}(x, \tau):=\int_{-\pi}^{+\pi} \hat{j}_{e}(k) d k$ are the macroscopic energy density and current, respectively. Let us split the current as

$$
j_{e}(x, \tau)=j_{e}^{<}\left(x, \tau ; k_{c}\right)+j_{e}^{>}\left(x, \tau ; k_{c}\right) ;
$$

we then consider $j_{e}^{<}=2 \int_{0}^{k_{c}} \hat{j}_{e}(k) d k \propto k_{c}^{2}$ - since $\hat{j}_{e}(k) \propto$ $k$ for $k \ll 1$, see Fig. 4 top left-and $j_{e}^{>}=-\kappa_{\text {kin }} \partial_{x} e$, with $k_{\text {kin }}>0$-i.e., Fick's law for the kinetic modes. Using the estimate of Eq. (8), $k_{c} \sim L^{-3 / 10}$, we obtain:

$$
\kappa_{e}^{<}\left(k_{c}\right) \propto L^{2 / 5}, \quad \kappa_{e}^{>}\left(k_{c}\right)=\kappa_{\text {kin }} .
$$

Plugging (13) into (12) and taking into account that in the bulk the energy associated to ballistic modes is constant, we obtain

$$
\partial_{x}\left[\kappa_{\text {kin }} \partial_{x} e^{>}\left(x ; k_{c}\right)\right]=0 .
$$

Imposing the fraction $1-k_{c} / \pi$ of kinetic modes to have "temperature" $T_{ \pm}$at the boundaries, and that the fraction of ballistic modes $k_{c} / \pi$ is at constant "temperature" $\bar{T}$, we have

$$
e(x)=e^{<}\left(x ; k_{c}\right)+e^{>}\left(x ; k_{c}\right)=\frac{k_{c}}{\pi} \bar{T}+\left(1-\frac{k_{c}}{\pi}\right) T(x),
$$

where $T(x)=T_{+}-(\Delta T / L) x$ is the Fourier profile [17], attained by the kinetic part as solution to (15). Thus, Eqs. (14)-(16) highlight how the kinetic modes dominate the energy density, despite the presence of an anomalous conduction.

Discussion.-Our numerical simulations show that the $\beta$-FPUT lattice does not thermalize locally; moreover, the scaling obtained for $\kappa_{e}$ is compatible with the law $L^{2 / 5}$. We have developed a theoretical argument, based on dimensional analysis of the kinetic equation, that supports this view. Because other numerical simulations and theoretical approaches have led to different exponents [14], we mention that the key ingredient in our approach is that the relaxation time, calculated from the collision integral, scales as $k^{-5 / 3}$ for small $k$. Such result is exact if computed at equilibrium, i.e., for a thermalized spectrum, hence it is subject to not necessarily universal corrections in nonequilibrium conditions. To verify its robustness, we have performed a direct numerical computation of the collision integral and we have calculated the relaxation timescale in the presence of different kind of perturbations, including also the introduction of a chemical potential. The results are contained in the Supplemental Material [41] and can be summarized as follows: for small perturbations, the $k^{-5 / 3}$ scaling is confirmed; however, as the perturbation grows, a departure from the $k^{-5 / 3}$ scaling, especially in the very low wave number region, is observed.

To conclude, strictly speaking, the $\beta$-FPUT lattice does not relax to local equilibrium, since only part of its normal modes reaches such a state. The system can be treated as made of two independent components, with distinct macroscopic behavior: the high- $k$ modes satisfying Fourier's law, with linear temperature profile and regular heat transport with finite heat conductivity, and the low- $k$ modes, scarcely interacting, carrying energy in a ballistic way (or close to ballistic). This differs in essence from heat conduction [45]. Our conclusions are in qualitative agreement with the efficient transport due to low frequency modes in carbon nanotubes, offering a simple first-principle interpretation to the persistence of coherent ballistically conducting modes observed experimentally at lengths up to more than $0.1 \mathrm{~mm}$ [46-48], about $10^{5}$ times the tube diameter and $10^{6}$ times the interatomic distance.

The authors thank Stefano Lepri, Joel Lebowitz, and Hong Zhao for helpful discussion. M. O., F. D. V., and 
G. D. have been funded by Progetto di Ricerca d'Ateneo CSTO160004, by the "Departments of Excellence 2018/ 202" Grant awarded by the Italian Ministry of Education, University and Research (MIUR) (No. L.232/2016). L. R. acknowledges partial support from MIUR Grant Dipartimenti di Eccellenza No. 2018-2022. M. O. and F. D. V. were supported by Simons Collaboration on Wave Turbulence, Grant No. 617006. G. D., M. O., and D. P. acknowledge the support of the EPSRC First Grant scheme (No. EP/P023770/1).

*Corresponding author. giovannidematteis@gmail.com

[1] C. Cercignani, Transp. Theory Stat. Phys. 2, 211 (1972).

[2] A. de Masi, R. Esposito, and J. Lebowitz, Commun. Pure Appl. Math. 42, 1189 (1989).

[3] R. Kubo, M. Yokota, and S. Nakajima, J. Phys. Soc. Jpn. 12, 1203 (1957).

[4] H. J. Kreuzer, Nonequilibrium Thermodynamics and Its Statistical Foundations (Oxford University Press, Oxford, 1981).

[5] H. B. Callen, Thermodynamics and an Introduction to Thermostatistics, 2nd ed. (Wiley, New York, 1998).

[6] Z. Rieder, J. Lebowitz, and E. Lieb, J. Math. Phys. (N.Y.) 8, 1073 (1967).

[7] S. Lepri, R. Livi, and A. Politi, Phys. Rep. 377, 1 (2003).

[8] A. Dhar, Adv. Phys. 57, 457 (2008).

[9] C.-W. Chang, D. Okawa, H. Garcia, A. Majumdar, and A. Zettl, Phys. Rev. Lett. 101, 075903 (2008).

[10] J. Chen, G. Zhang, and B. Li, Nano Lett. 10, 3978 (2010).

[11] S. Shen, A. Henry, J. Tong, R. Zheng, and G. Chen, Nat. Nanotechnol. 5, 251 (2010).

[12] N. Yang, G. Zhang, and B. Li, Nano Today 5, 85 (2010).

[13] S. Liu, X. Xu, R. Xie, G. Zhang, and B. Li, Eur. Phys. J. B 85, 337 (2012).

[14] Thermal Transport in Low Dimensions: From Statistical Physics to Nanoscale Heat Transfer, edited by S. Lepri (Springer, Heidelberg, 2016), Vol. 921.

[15] S. Nazarenko, Wave Turbulence (Springer Science \& Business Media, Berlin-Heidelberg, 2011), Vol. 825.

[16] A. Pereverzev, Phys. Rev. E 68, 056124 (2003).

[17] H. Spohn, J. Stat. Phys. 124, 1041 (2006).

[18] J. Lukkarinen, in Thermal Transport in Low Dimensions (Springer, Heidelberg, 2016), pp. 159-214.

[19] M. Onorato, L. Vozella, D. Proment, and Y. V. Lvov, Proc. Natl. Acad. Sci. U.S.A. 112, 4208 (2015).

[20] Y. V. Lvov and M. Onorato, Phys. Rev. Lett. 120, 144301 (2018).

[21] L. Pistone, M. Onorato, and S. Chibbaro, Europhys. Lett. 121, 44003 (2018).

[22] L. Pistone, S. Chibbaro, M. D. Bustamante, Y. V. Lvov, and M. Onorato, Math. Biosci. Eng. 1, 672 (2019).
[23] K. Aoki, J. Lukkarinen, and H. Spohn, J. Stat. Phys. 124, 1105 (2006).

[24] S. Lepri, R. Livi, and A. Politi, Europhys. Lett. 43, 271 (1998).

[25] T. Prosen and D. K. Campbell, Phys. Rev. Lett. 84, 2857 (2000).

[26] J. Lukkarinen and H. Spohn, Commun. Pure Appl. Math. A 61, 1753 (2008).

[27] K. Aoki and D. Kusnezov, Phys. Lett. A 309, 377 (2003).

[28] S. Lepri, P. Sandri, and A. Politi, Eur. Phys. J. B 47, 549 (2005).

[29] P. I. Hurtado, Phys. Rev. Lett. 96, 010601 (2006).

[30] C. Giberti and L. Rondoni, Phys. Rev. E 83, 041115 (2011).

[31] C. Giberti, L. Rondoni, and C. Vernia,arXiv:1706.02886.

[32] C. Giberti, L. Rondoni, and C. Vernia, Eur. Phys. J. Special Topics 228, 129 (2019).

[33] C. Mejía-Monasterio, A. Politi, and L. Rondoni, Phys. Rev. E 100, 032139 (2019).

[34] E. Fermi, J. Pasta, S. Ulam, and E. Fermi, Los Alamos,. Report No. LA-1940, in Collected Papers 2, 977 (1955).

[35] The Fermi-Pasta-Ulam Problem: A Status Report, edited by G. Gallavotti (Springer, Berlin-Heidelberg, 2007), Vol. 728.

[36] T. Dauxois, Phys. Today 61, No. 1, 55 (2008).

[37] S. Lepri, R. Livi, and A. Politi, Chaos 15, 015118 (2005).

[38] M. D. Bustamante, K. Hutchinson, Y. V. Lvov, and M. Onorato, Commun. Nonlinear Sci. Numer. Simul. 73, 437 (2019).

[39] L. Saint-Raymond, Hydrodynamic Limits of the Boltzmann Equation (Springer Science \& Business Media, BerlinHeidelberg, 2009).

[40] A. Dhar and K. Saito, in Thermal Transport in Low Dimensions (Springer, Heidelberg, 2016), pp. 39-105.

[41] See the Supplemental Material at http://link.aps.org/ supplemental/10.1103/PhysRevLett.125.024101 for the numerical computation of the collision integral and a detailed discussion of the results, which includes Refs. [42-44].

[42] J. M. O. De Zarate and J. V. Sengers, Hydrodynamic Fluctuations in Fluids and Fluid Mixtures (Elsevier, Amsterdam, 2006).

[43] L. Conti, P. De Gregorio, G. Karapetyan, C. Lazzaro, M. Pegoraro, M. Bonaldi, and L. Rondoni, J. Stat. Mech. (2013) P12003.

[44] M. Baiesi, C. Burigana, L. Conti, G. Falasco, C. Maes, L. Rondoni, and T. Trombetti, Phys. Rev. Research 2, 013210 (2020).

[45] S. Chen, Y. Zhang, J. Wang, and H. Zhao, Phys. Rev. E 87, 032153 (2013).

[46] D. Donadio, in Thermal Transport in Low Dimensions (Springer, Heidelberg, 2016), pp. 275-304.

[47] X. Zhang, M. Hu, and D. Poulikakos, Nano Lett. 12, 3410 (2012).

[48] C.-W. Chang, in Thermal Transport in Low Dimensions (Springer, Heidelberg, 2016), pp. 305-338. 